\title{
Persistent upregulation of the $\beta$-tubulin tubb6, linked to muscle regeneration, is a source of microtubule disorganization in dystrophic muscle
}

\author{
Davide Randazzo ${ }^{1}$, Umara Khalique ${ }^{1}$, Joseph J. Belanto ${ }^{2}$, Aster Kenea ${ }^{1}$, \\ Dana M. Talsness ${ }^{2}$, John T. Olthoff ${ }^{2}$, Michelle D. Tran ${ }^{1}$, Kristien J. Zaal ${ }^{1}$, \\ Katherine Pak ${ }^{3}$, Iago Pinal-Fernandez ${ }^{3,4}$, Andrew L. Mammen ${ }^{3,4}$, \\ Dan Sackett ${ }^{5}$, James M. Ervasti ${ }^{2}$ and Evelyn Ralston ${ }^{1, *}$
}

\begin{abstract}
${ }^{1}$ Light Imaging Section, Office of Science and Technology, National Institute of Arthritis and Musculoskeletal and Skin Diseases (NIAMS), National Institutes of Health (NIH), Bethesda, MD, USA, ${ }^{2}$ Department of Biochemistry, Molecular Biology, and Biophysics, and Program in Molecular, Cellular, Developmental Biology, and Genetics, University of Minnesota-Twin Cities, Minneapolis, MN, USA, ${ }^{3}$ Laboratory of Muscle Stem Cells and Gene Regulation, Muscle Disease Unit, NIAMS, NIH, Bethesda, MD, USA, ${ }^{4}$ Johns Hopkins University School of Medicine, Baltimore, MD, USA and ${ }^{5}$ Eunice Kennedy Shriver National Institute of Child Health and Human Development (NICHD), NIH, Bethesda, MD, USA
\end{abstract}

\footnotetext{
*To whom correspondence should be addressed at: Light Imaging Section, Office of Science and Technology, National Institute of Arthritis and Musculoskeletal and Skin Diseases, National Institutes of Health 9000 Rockville Pike, Bldg. 50, Rm. 1535, Bethesda, MD 20892-8023, USA. Tel: +1-301-496-6164; Fax: +1-301-402-3417; Email: evelyn.ralston@nih.gov
}

Human Molecular Genetics, 2019, 28(7), 1117-1135 doi: $10.1093 / \mathrm{hmg} / \mathrm{ddy} 418$.

The authors would like to apologize for errors within the legend of Figure 10. The legend has been corrected online and in print as below:

"2.300.30-fold" changed to " $2.30 \pm 0.30$-fold"

"4.100.41-fold" changed to " $4.10 \pm 0.41$-fold"

"1.460.05-fold" changed to " $1.46 \pm 0.05$-fold" 